\title{
High performance electrochemical biosensor based on 3D nitrogen-doped reduced graphene oxide electrode and tetrahedral DNA nanostructure
}

Rong Tian, Weihua Ning, Minghai Chen, Cheng Zhang, Qingwen Li and J ingwei Bai

The self-archived postprint version of this journal article is available at Linköping University Institutional Repository (DiVA):

http:/ / urn.kb.se/ resolve?urn=urn:nbn:se:liu:diva-154541

N.B.: When citing this work, cite the original publication.

Tian, R., Ning, W., Chen, M., Zhang, C., Li, Q., Bai, J ., (2019), High performance electrochemical biosensor based on 3D nitrogen-doped reduced graphene oxide electrode and tetrahedral DNA nanostructure, Talanta, 194, 273-281. https:// doi.org/ 10.1016/j.talanta.2018.09.110

Original publication available at:

https:// doi.org/ 10.1016/j.talanta.2018.09.110

Copyright: Elsevier

http:// www.elsevier.com/

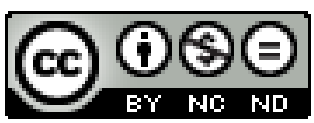




\title{
High performance electrochemical biosensor based on 3D nitrogen-doped reduced graphene oxide electrode and tetrahedral DNA nanostructure
}

Rong Tian ${ }^{\mathrm{a}, \mathrm{b}}$, Weihua Ning ${ }^{\mathrm{c}, \mathrm{d}}$, Minghai Chen ${ }^{\mathrm{b}}$, Cheng Zhang ${ }^{\mathrm{e}}$, Qingwen Li ${ }^{\mathrm{b} *}$, Jingwei $\mathrm{Bai}^{\mathrm{a} *}$

a School of Pharmaceutical Sciences of Tsinghua University and Tsinghua-Peking Joint Center for Life Sciences, Beijing, China 100084

b Division of Advanced Nanomaterials and Key Lab of Nanodevices and Applications, Suzhou Institute of Nano-Tech and Nano-Bionics, Chinese Academy of Sciences, Suzhou 215123, PR China

c Department of Physics, Chemistry, and Biology, Linköpings Universitet, Linköping SE-581 83, Sweden

d Key Lab for Flexible Electronics \& Institute of Advanced Materials, Jiangsu National Synergistic Innovation Center for Advanced Materials (SICAM), Nanjing Tech University, 30 South Puzhu Road, Nanjing, 211816 PR China

e Institute of Software, School of Electronics Engineering and Computer Science, Peking University. Key laboratory of High Confidence Software Technologies, Ministry of Education, Beijing 100871, China

* Corresponding author: Fax: +86 10 62789815, +86 51262872577

E-mail address: jingwbai@mail.tsinghua.edu.cn(J. Bai) qwli2007@sinano.ac.cn (Q. Li)

\begin{abstract}
In this study, an electrochemical biosensor was developed for highly sensitive and specific detection of target miRNA-155. The structure was formed by the hybridization of a tetrahedral DNA nanostructure-based biomolecular probe assembled on 3D nitrogen-doped reduced graphene oxide/ gold nanoparticles (3D N-doped rGO/AuNPs) electrode surface. Upon addition of target miRNA-155, the gold and silver nanorod/ thionine/ complementary DNA (AuAgNR/Thi/F) was
\end{abstract}


hybridized with the target, and used for signal amplification, catalyzing the reduction of Thi as an electron mediator. Due to the signal amplification by the enhanced immobilization of DNA on the surface of 3D N-doped rGO/AuNPs electrode and AuAgNR/Thi, coupling the low background signal produced by blank solution, electrochemical performance of the device was optimized to be proportional to miRNA-155 concentration in the range of $1 \times 10^{-11}$ to $1 \times 10^{-4} \mathrm{M}$ with a detection limit of $1 \times 10^{-12} \mathrm{M}$. In addition, direct detection in serum is demonstrated with high specificity. Thus, this biosensor is potentially applicable for microRNA detection in medical research and early clinical diagnosis.

Keywords: 3D N-doped rGO/AuNPs, MicroRNA, AuAgNR, Tetrahedral DNA, Electrochemical biosensor

\section{Introduction}

MicroRNAs (miRNAs) are a group of small (approximately 22 nucleotides), endogenous, and non-coding RNA molecules, which regulate the expression of target genes [1]. Recently, accumulative evidence indicates that miRNAs are highly correlated with cancer initiation, tumorigenesis and cell differentiation [2-4]. Thus, miRNAs have been regarded as biomarkers and therapeutic targets in cancer diagnosis and treatment. Analytical approaches based on optical methods have been developed for detection of miRNAs, such as microarray and surface plasmon resonance [5, 6]. However, these methods are typically time-consuming, require precise instrumentation and are less accurate, thus hindering their wide spread application in clinic diagnosis. Sensor assays have drawn much attention for analysis due to their high sensitivity, simplicity and short turn-around time [7-12]. For example, Turner's group developed a highly specific biotinylated DNA/LNA molecular beacon (MB) probe conjugated with gold nanoparticles (AuNPs) to create an integrated, dual function bio-label (biotin-MB-AuNPs) for both biorecognition, signal generation for the simple and sensitive detection of miRNA-21[13]. Zhang's group developed an electrochemical immunosensor for tumor marker detection with a redox-catalysis 'all-in-one' mechanism [14]. Liu's group realized the electrochemical detection of 
miRNAs based on the signal amplification of AuNPs, enzymes and redox-cycling [15]. Zhang's group used enzyme-free mismatched CHA for the first time and exhibited excellent analytical performance towards miRNAs determination [16]. Most of current electrochemical biosensors for miRNAs suffer from poor stability, and the sensitivity needs to be further improved for early stage diagnosis.

A typical way to improving the sensitivity of electrochemical biosensor is to engineer the electrode with large surface-volume ratio, together with improved electrochemical activity. Recent development in electrochemically active 2D materials paves the way for highly sensitive sensor electrodes. For example, nitrogen-doped graphene with enhanced electrochemical activity is considered as an ideal electrochemical material for energy applications such as electrocatalysis and supercapacitors [17-20]. Unfortunately, directly applying nitrogen-doped grapheme to biological sensing applications didn't yield a superior performance, because the material itself does not provide catalytic specificity to the particular oxidation/reduction process. This problem can be resolved by introducing metal nanoparticles to accelerate the electron transfer between the electrode surface and modifiers, and further amplify the electrical response. In this way, AuAg bimetallic NPs can facilitate redox processes because of their superior electrochemical properties [21]. AuAgNRs were used to realize the signal amplification due to a localized electric field enhancement in the core-shell structure, which can electrostatically adsorbed onto the negatively charged surface of DNA.

Supramolecular chemistry started and developed as the chemistry of the entities generated via intermolecular non-covalent interactions. It becames progressively the chemistry of molecular information, involving the storage of information at the molecular level [22, 23]. It has been widely used in sensing, catalysis and biology because of their unusual properties. The chemosensing ensemble strategy uses a supramolecular assembly with a competitive assay, in which it works in the same way as that of antibody-based immunoassay. Similarly, the supramolecular ensemble in such assays uses a receptor, designed for competitive complexation with a guest species, together with an indicator to sense presence of the ions/neutral analyte. 
An important aspect of high performance electrochemical biosensors for nucleic acids, relies on careful design of the nucleic acid binding probe, considering the difficulty of controlling the probe orientation and density on the electrode surface. Although single stranded DNA and hairpin DNA have been reported as capture probes, nonspecific interactions, steric hindrance and entanglement between the probes, have negative effects on the selectivity and reproducibility [24, 25]. Herein, we firstly reported an ultrasensitive electrochemical detection of miRNA-155, by coupling the signal amplification strategy of 3D N-doped rGO/AuNPs electrode and AuAgNR/Thi/F as a recognition element. The characteristics consisted in this assay protocol mainly including (1) the N-doped rGO was selected as the combination of $\mathrm{Au}$ because of its relatively higher abundance and lower cost, and 3D N-doped rGO/AuNPs also has high electronic conductivity, which could accelerate response time and widen linear range; (2) The thiolated DNA tetrahedral nanostructures binding to the AuNPs surface provides a solution-like environment, increases biomolecule target accessibility and reduces the surface crowding effect, hence, greatly enhancing the electrochemical miRNA biosensor's sensitivity and stability; (3) AuAgNR are chosen as the supporting materials for DNA and Thi to enhance the electrochemical signals. We applied this biosensor scheme for the detection of miRNA-155. The combination of the above advantages resulted in high sensitivity, excellent selectivity and good stability for the detection of the target miRNA. 


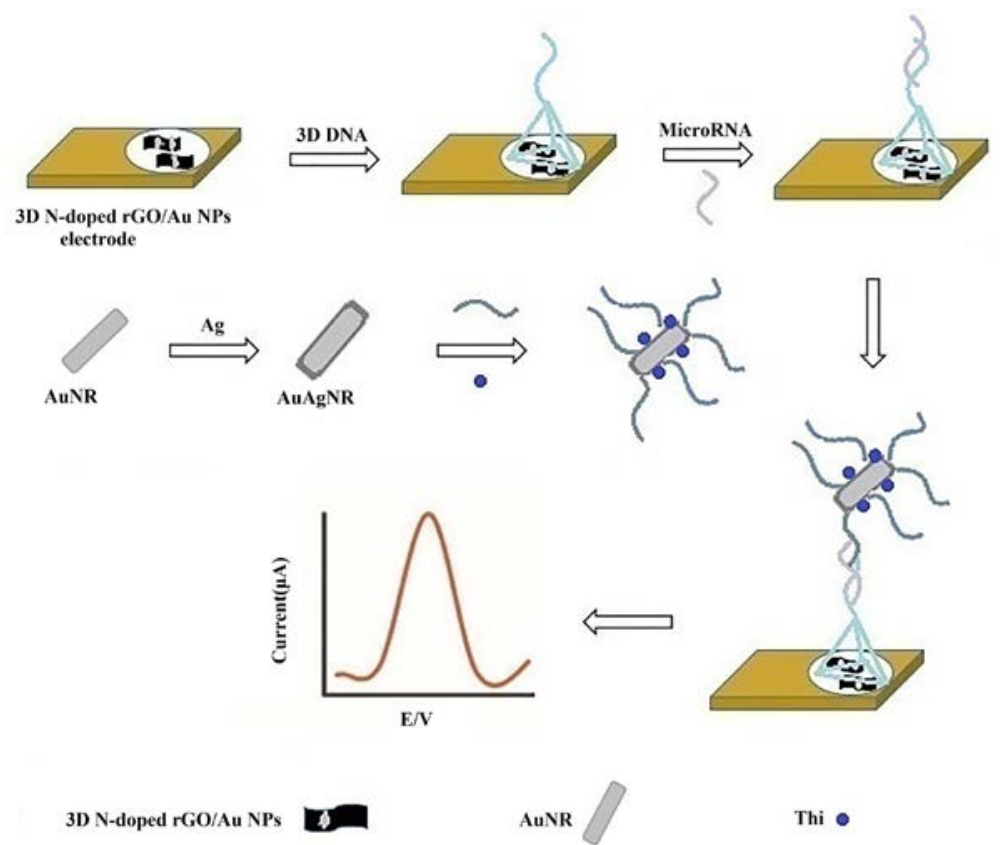

Scheme 1 Schematic diagram for the electrochemical detection of miRNA-155

\section{Materials and methods}

\subsection{Chemicals and reagents}

Sodium borohydride $\left(\mathrm{NaBH}_{4}\right)$ and silver nitrate $\left(\mathrm{AgNO}_{3}\right)$ were purchased from Sigma Aldrich (USA). Hydrogen tetrachloroaurate (III) trihydrate $\left(\mathrm{HAuCl}_{4} \cdot 3 \mathrm{H}_{2} \mathrm{O}\right)$, graphene oxide (GO), DL-aspartic acid, thionine (Thi), cetyltrimethyl ammonium bromide (CTAB) and ascorbic acid (AA) were purchased from $\mathrm{J}$ \& $\mathrm{K}$ Chemical Technology (Beijing, China). Uric acid was purchased from Sigma Aldrich (St. Louis, MO). Potassium ferricyanide $\left(\mathrm{K}_{3}\left[\mathrm{Fe}(\mathrm{CN})_{6}\right]\right)$, potassium ferrocyanide $\left(\mathrm{K}_{4}\left[\mathrm{Fe}(\mathrm{CN})_{6}\right]\right)$ and potassium chloride (KCl) were purchased from Linfeng Chemical Reagent Co., Ltd (Shanghai, China). The buffers and solutions involved in this research were made as follows: DNA immobilization buffer ( $300 \mathrm{mM} \mathrm{NaCl,} 25 \mathrm{mM}$ tris-acetate, $\mathrm{pH}$ 8.0) and the supporting electrolyte phosphate buffer saline (PBS, $0.1 \mathrm{M})$. DNA and miRNAs sequences were synthesized by Sangon Biological Engineering Technology Co. Ltd. (Shanghai, China) and the detail oligonucleotide sequences are listed in Table 1. Other reagents were of analytical grade and used without further purification. Double distilled water was used throughout the experiments.

Table 1 Synthetic sequences used in the experiments 
ATGATACCGCCGAGAAGAGCACATCGTTCGACATTACAAAGTCTGAAT

A CCTTACA $\mathrm{T}_{10}$ ATCACGA

B

HS- C $_{6}$-CATAACCTGGGAGCGTAGATAATGTCGAACGATGTGACAGTTG ACGGACCACTAT

HS- C $_{6}$-CTTCTCGGCGGTATCATCTAAGGGTGCATCACAGCAAAATAGT

C GGTCCGTCAACT

HS-

$\mathrm{D}$

C6-TACGCTCCCAGGTTATGTTTGCTGTGATGCACCCTTCGTGTAAG

E

GATTCAGACTT

UUAAUGCUAAUCGUGAUAGGGG

F HS-TTAGCATTAATTTT

G AGUUGUAGUCAGACUAUUCGAU

$\mathrm{H}$ TACACCTTTTAGAGTACGTCA

I GGGGAUAGUGGUAAUCGUAAUU

E(miRNA-155), F(complementary), G(miRNA-21),H(non-complementary)

I(single-base mismatched microRNA sequence)

\subsection{Characterization}

The particle size and morphology of the resulting products were studied by transmission electron microscopy (TEM) (Tecnai G2 F20 S-Twin, FEI, USA) and scanning electron microscopy (SEM) (Quanta 400 FEG, FEI, USA) with Apollo 40 SDD X-ray Energy Dispersive Spectrometer (EDS). Fourier transform infrared (FT-IR) spectroscopic measurements (Vector 22, Bruker) using $\mathrm{KBr}$ pressed disks was used. Ultraviolet visible spectroscopy (UV-is) was recorded on an Agilent UV-is spectrophotometer. The powder X-ray diffraction (XRD) characterization was performed using X-ray diffraction (Bruker, D8 Focus, Karlsruhe, Germany) with $\mathrm{Cu}$ $\mathrm{K}$ radiation at room temperature. The electrochemical performance of the samples was characterized in a three-electrode test system by using a CHI 660 electrochemical workstation (Chenhua, Shanghai).

\subsection{Preparation of 3D N-doped rGO/AuNPs and AuAgNR/Thi/F}

The purchased GO was used as raw material for the synthesis of 3D N-doped 
rGO [26]. $0.15 \mathrm{~g}$ of amino acid was dissolved in $30 \mathrm{~mL}$ of $2 \mathrm{mg} \mathrm{mL}^{-1}$ aqueous GO solution (the mass ratio of amino acid to GO was 2.5:1). The mixture was magnetically stirred for 10 minutes, then transferred to a teflon-lined autoclave and subjected to hydrothermal treatment for $3 \mathrm{~h}$ at $160{ }^{\circ} \mathrm{C}$. After that, the autoclave was naturally cooled to room temperature and the as-prepared 3D N-doped rGO was removed from the autoclave and immersed in deionized water for seven days, deionized water was renewed once a day to remove any unreacted reagent. The 3D $\mathrm{N}$-doped rGO was freeze-dried under vacuum for further characterization. The final electrode was prepared by electrodeposition at $-1.5 \mathrm{~V} \sim 0.6 \mathrm{~V}(50 \mathrm{mV} / \mathrm{s})$ for 10 cycles in a mixture of $15 \mathrm{~mL} 3 \mathrm{D}$ N-doped rGO with $1.0 \mathrm{mM} \mathrm{HAuCl}_{4}$ in $\mathrm{pH} 7 \mathrm{PBS}$.

\subsection{Synthesis of AuAgNR/Thi/F}

AuNRs were prepared according to the seed-mediated growth method optimized by Nikoobakht and El-Sayed [27]. Briefly, a seed solution was prepared by mixing of $5 \mathrm{~mL}$ of $\mathrm{HAuCl}_{4}(0.5 \mathrm{mM}), 5 \mathrm{~mL}$ of CTAB $(0.1 \mathrm{M})$ and $0.6 \mathrm{~mL}$ of freshly prepared 10 $\mathrm{mM}$ ice-cold sodium borohydride $\left(\mathrm{NaBH}_{4}\right)$, then stirred till the solution color changing from yellow to brown. The synthesis of AuNRs was then initiated by mixing $0.25 \mathrm{~mL}$ of $4 \mathrm{mM} \mathrm{AgNO} 3,5 \mathrm{~mL}$ of $1.0 \mathrm{mM} \mathrm{HAuCl}_{4}$ and $5 \mathrm{~mL}$ of $0.1 \mathrm{M} \mathrm{CTAB}$ solution. Then, $0.06 \mathrm{~mL}$ of $0.1 \mathrm{M} \mathrm{AA}$ was added to reduce the gold ions. With continuously stirring, $12 \mathrm{~mL}$ of the seed solution was added and the solution was aged for $20 \mathrm{~h}$ to ensure full growth of AuNRs. Finally, AuNRs were centrifuged and washed for 3 times. In order to form AuAg alloyed nanorods, a quantity of $0.4 \mathrm{~mL}$ AuNRs solution was redispersed in $4 \mathrm{~mL}$ of $1 \mathrm{wt} \%$ PVP aqueous solution. Then, 0.5 $\mathrm{mL}$ of $1.0 \mathrm{mM} \mathrm{AgNO}_{3}$ and $0.1 \mathrm{~mL}$ of $0.1 \mathrm{M}$ ascorbic acid were added with gently mixing. At this stage, $\operatorname{Ag}(\mathrm{I})$ was not reduced due to the relatively low $\mathrm{pH}$ of the solution. The reaction was started by injecting $0.4 \mathrm{~mL}$ of $0.1 \mathrm{M} \mathrm{NaOH}$ solution, followed by quickly mixing for two minutes to obtain the AuAgNRs. The obtained product was added into Thi and F mixing solution for strirring $12 \mathrm{~h}$.

\subsection{Fabrication of the miRNA biosensor}

Tetrahedral DNA probe was prepared following the literature report [28]. 3D $\mathrm{N}$-doped rGO/AuNPs was incubated in $6 \mu \mathrm{M}$ tetrahedral DNA probes for $12 \mathrm{~h}$ at 37 
${ }^{\circ} \mathrm{C}$ in a homo-thermal water bath chamber. Different concentrations of miRNA-155 were added to the solution and kept at $37^{\circ} \mathrm{C}$ for $1 \mathrm{~h}$. Finally, $10 \mu \mathrm{L}$ of AuAgNR/Thi/F dispersion was drop-wise added to the surface of the 3D N-doped rGO/AuNPs/ tetrahedral DNA electrode and dried at $37^{\circ} \mathrm{C}$ for $2 \mathrm{~h}$. The final electrodes were washed with PBS for three times before use.

\section{Results and discussion}

\subsection{Characterization of nanomaterials}

Apart from excellent biocompatibility, 3D structure is a good candidate for fabricating biosensors, because its large surface area can increase significantly detection signals. Here, a 3D N-doped rGO/AuNPs electrode was prepared through electro-controlled co-reduction method. To our knowledge, there are no previous reports on the preparation of 3D N-doped rGO/AuNPs film by this technique. 3D N-doped rGO and AuNPs were simultaneously electro-deposited at a potential of -1.5 $\mathrm{V} \sim 0.6 \mathrm{~V}$. The SEM images show that GO (Fig. 1A) and 3D N-doped rGO prepared by adding DL-aspartic acid (Fig. 1B). By adding DL-aspartic acid, GO was reduced to form a stacked layers rGO matrix. And uniform AuNPs were visible in the 3D N-doped rGO samples.

FT-IR spectra was employed to identify the functional groups and investigate the reduction process. As shown in curve a of Fig. 2, other than characteristic $\mathrm{sp}^{2}$ hybridized carbon skeleton vibration at $1562 \mathrm{~cm}^{-1}$ [29], the peak at $1714 \mathrm{~cm}^{-1}$ can be assigned to a strong $\mathrm{C}=\mathrm{O}$ stretch [30] and $\mathrm{C}-\mathrm{OH}$ stretch can be found at $1114 \mathrm{~cm}^{-1}$ indicating oxygen containing groups, such as hydroxyl, epoxy and carboxyl groups. After treatment with DL-aspartic acid (curve b), the peak at $1714 \mathrm{~cm}^{-1}$ for $\mathrm{C}=\mathrm{O}$ stretch is vanished, the new peaks such as the $\mathrm{C}-\mathrm{N}$ stretching vibration band at 1406 $\mathrm{cm}^{-1}$ and the typical $\mathrm{C}=\mathrm{N}$ stretching vibration band at $1315 \mathrm{~cm}^{-1}$ appears, which are correspond to the characteristic bands of N-doping [31]. Compared with GO and 3D $\mathrm{N}$-doped rGO, some group adsorption peaks of GO were changed, implying that 3D $\mathrm{N}$-doped rGO was formed.

To obtain the further crystallographic information of the 3D N-doped rGO, X-ray 
powder diffraction (XRD) spectra was conducted. XRD measurement for the GO and 3D N-doped rGO are shown in Fig. 2B. For the sample of GO, the strong diffraction peak at $2 \theta=10.2^{\circ}$ can be indexed as the GO (002) peak. The introduced oxygen-containing functional groups on graphite sheet layer and the interlayer of hydrone result in $\mathrm{d}=0.866 \mathrm{~nm}$ which is larger than graphite of $\mathrm{d}=0.334 \mathrm{~nm}$. After hydrothermal reaction, the (002) peak of GO shifted to $2 \theta=13.4^{\circ}$, indicating that the graphite lattice structure has been restored to a certain extent. With N-doped, the (002) peak of 3D N-doped rGO was observed at $2 \theta=27.1^{\circ}$ [32]. The EDS analysis further proof the existence of $\mathrm{N}$ element, indicating that the atomic ratio of $3 \mathrm{D} \mathrm{N}$-doped rGO/AuNPs (Fig. 2C) and revealing the successful synthesis of 3D N-doped rGO/AuNPs under proposed conditions.

Highly sensitive bio-assays have been usually found using labeled detection, and the label is frequently an enzyme which can generate an electroactive species in the presence of substrate. Although such approaches provide high sensitivity, the deterioration of enzyme activity over time can be problematic. Alternatively, metal nanoparticles or redox mediators can provide labels of greater stability. Here, AuAgNR were used as ideal carriers to attach Thi redox molecules through the Ag-S stacking adsorption between them. Fig. 3 A and B show the TEM images of the original AuNR and AuAgNR. The contrast difference surround AuAgNR indicated shell formation after $\mathrm{Ag}^{+}$reduction due to surface rearrangement and developing crystal defects. This is also verified by UV-Vis obsorption. UV-Vis absorption spectra were used to examine how the silver coating affects the surface plasmon resonance of the AuNRs. As the longitudinal surface plasmon mode of AuNRs peaked at 525 and $675 \mathrm{~nm}$ blue shift, the result was similar with the result of literature [33-35].The blue-shift can arise from two effects. First, the dielectric function of silver is different from gold, therefore the effective dielectric function varies for silver coating, and second, a homogeneous layer coating of a rod lowers the overall aspect ratio. FTIR experiments were carried out to further demonstrate the formation of AuAgNR/Thi/F bioconjugates (Fig. 3D). In the spectrum of AuAgNR/Thi/F (curve c), the characteristic bands for Thi $\left(1495 \mathrm{~cm}^{-1}\right)$ and $\mathrm{F}\left(1660 \mathrm{~cm}^{-1}\right.$ and $\left.1225 \mathrm{~cm}^{-1}\right)$ appear, but 
AuNR (curve a) and AuAgNR (curve b) are no obvious peaks, indicating successful assembly of the Thi and DNA on the nanoprobes [36]. Fig. 3E illustrated the XRD patterns of the AuAgNR and AuAgNR/Thi/F, respectively. For AuAgNR (red line) and AuAgNR/Thi/F (black line), four additional peaks at 38.4, 44.5, 65.1, and 78 were also observed, which were resulted from the (111), (200), (220) and (311) planes of Au (JCPDS card No. 04-0784) and Ag (JCPDS card No. 04-0783)[37]. Meanwhile, AuAgNR/Thi/F also exhibited a similar XRD pattern to that of AuAgNR, indicating the loading of Thi and F onto AuAgNR didn't impact the crystalline integrity of AuAgNR.

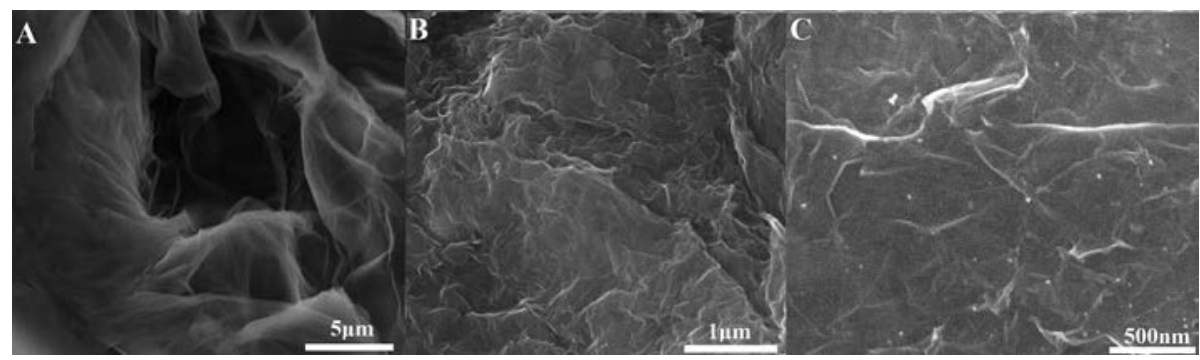

Fig. 1 SEM images of GO (A), 3D N-doped rGO (B) and 3D N-doped rGO/AuNPs (C)
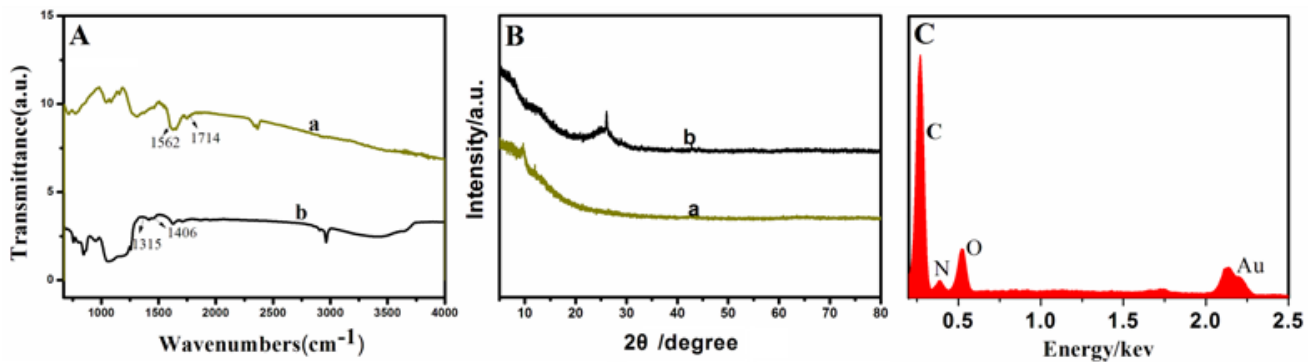

Fig. 2 (A) FT-IR spectra, (B) XRD patterns of GO (a) and 3D N-doped rGO (b); (C) EDS pattern of 3D N-doped rGO/AuNPs 

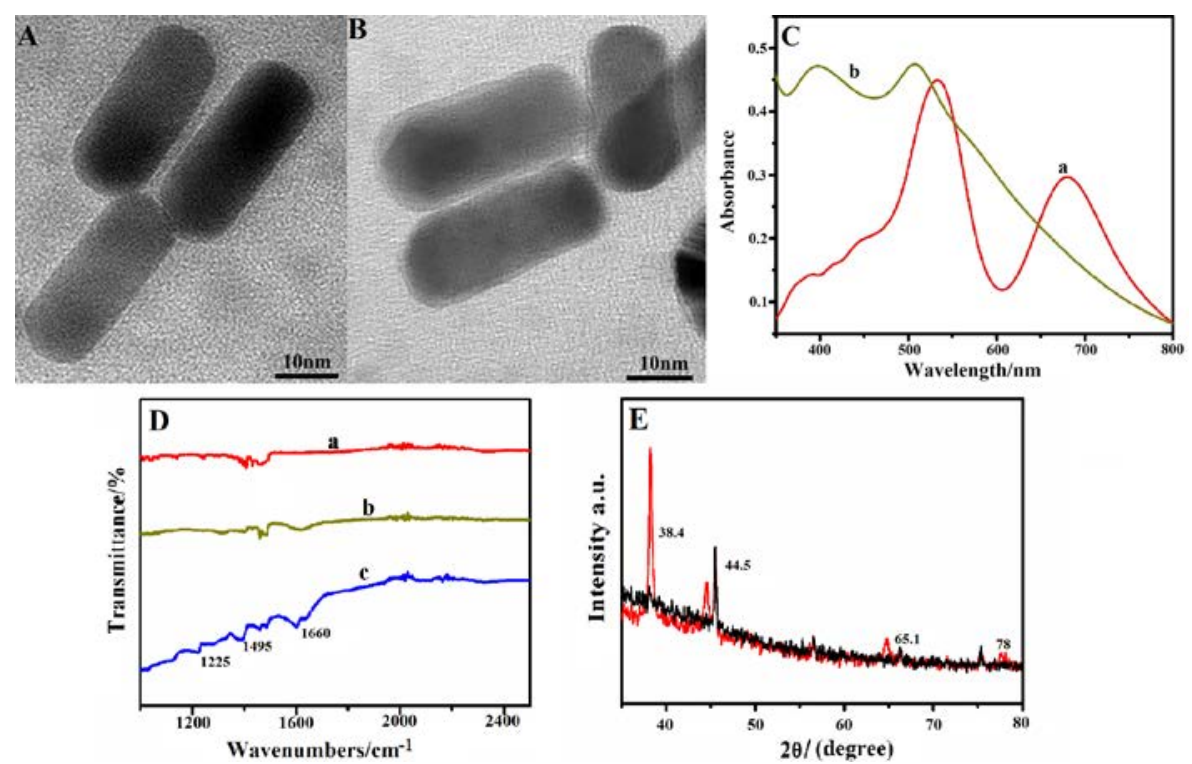

Fig. 3 SEM images of AuNR (A), AuAgNR (B), UV-Vis absorption spectra of AuNR (a), AuAgNR (b) (C), FTIR spectra of AuNR(a), AuAgNR(b), AuAgNR/Thi/F(c)（D) , XRD pattern of AuAgNR (red line) and AuAgNR/Thi/F (black line). The region of $2 \theta$ is from 35 to $80^{\circ}(\mathrm{E})$

\subsection{Characterization of the biosensor fabrication process}

The mechanism of this microRNA sensing method is illustrated. Generally, tetrahedral DNA is formed by four single stranded DNA. The four single stranded DNA (tetrahedron A, B, C, D) are engineered to form the six edges of the tetrahedral nanostructure. Three thiol vertices of the tetrahedron facilitate the modification of the tetrahedral DNA on the 3D N-doped rGO/AuNPs electrode surface, which reinforces the molecular recognition efficiency. In the presence of microRNA, AuAgNR/Thi/F was connected with tetrahedral DNA on the 3D N-doped rGO/AuNPs electrode. Fig.4 shows the successful formation of tetrahedral DNA probes was confirmed by polyacrylamide gel electrophoresis (PAGE). The bioactivity and amount of immobilized oligonucleotide can be significantly affected by the surface properties of the transducer.

The modification process of the 3D N-doped rGO/AuNPs film surface was characterized by $\mathrm{CV}$ of the 3D N-doped rGO/AuNPs, tetrahedral DNA, miRNA-155 and AuAgNR/Thi/F modified electrodes (Fig. 5A), as a result of sequential reduction of peak currents and the increase of peak-to-peak separation due to the binding of the DNA component. Fig. 5B shows the Nyquist plots of impedance spectra at different 
electrode surfaces in $0.1 \mathrm{M} \mathrm{KCl}$ solution containing $5 \mathrm{mM} \mathrm{Fe}(\mathrm{CN})_{6}{ }^{3-/ 4}$. To obtain more information from EIS results, the working electrode was modeled by the equivalent circuit, which was used to fit the EIS data. The change in the diameter of the semicircle reflected the change in the interfacial charge-transfer resistance $\left(R_{c t}\right)$. In addition to $R_{c t}$, the equivalent circuit contains $R_{s}$ that is the electrolyte solution resistance, the Warburg impedance $\mathrm{Z}_{\mathrm{w}}$ and the constant phase element related to double layer capacitance $\mathrm{C}_{\mathrm{dl}}$. For the 3D N-doped rGO/AuNPs film (curve a), the value of $R_{c t}$ was $700 \Omega$, which indicated that the electrons on the surface of the electrode transport rapidly. After the immobilization of tetrahedral DNA, the value of $\mathrm{R}_{\mathrm{ct}}$ increased to $2498 \Omega$, attributing to the fact that negative charges of the DNA phosphate backbone prevent redox of $\mathrm{Fe}(\mathrm{CN})_{6}{ }^{3-/ 4-}$ approaching the electrode surface. When miRNA-155 (curve c) was modified on 3D N-doped rGO/AuNPs, the $\mathrm{R}_{\mathrm{ct}}$ values increased dramatically to $3298 \Omega$. The combination of AuAgNR/Thi/F did not cause obvious change in the $R_{c t}$ value (curve $d, 3330 \Omega$ ), owing to the good conductivity of AuAgNR. These EIS results were in good agreement with the CVs results.

As shown in Fig. 6A, the sensor incubating with tetrahedral DNA showed a clear increase in the electrochemical signal compared to the sensor incubating without tetrahedral DNA, because tetrahedral DNA could connect the miRNA-155 for the combination of AuAgNR/Thi/F. Additionally, the electrochemical signal increased when the DNA sensor was incubated with AuAgNR/Thi/F compared to the AuAgNR/Thi and AuAgNR/F (Fig. 6B), owing to the fact that F can connect the miRNA-155 for amplifying the signal and the reduction of Thi. This interesting phenomenon indicated that the DNA sensor was incubated with AuAgNR/Thi/F have the most efficient catalytic ability, the probable reason was that the cooperative ability of Thi and F. The comparison of the all results demonstrates that our proposed electrochemical DNA sensor could significantly improves the detection sensitivity. Electrode surface modification is characterized by LSV. As depicted in Fig. 6C, no obvious peak appears (curve a). DNA tetrahedron modified electrode (curve b) and microRNA (curve c) cannot exhibit a significant current peak unless the incubation 
with AuAgNR/ F (curve d) demonstrating the fact that microRNA can effectively capture AuAgNR/F on the electrode surface.

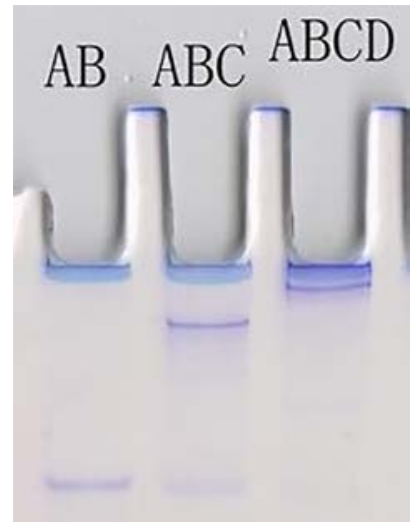

Fig.4 Gel electrophoretic analysis of the formation of tetrahedral DNA probes
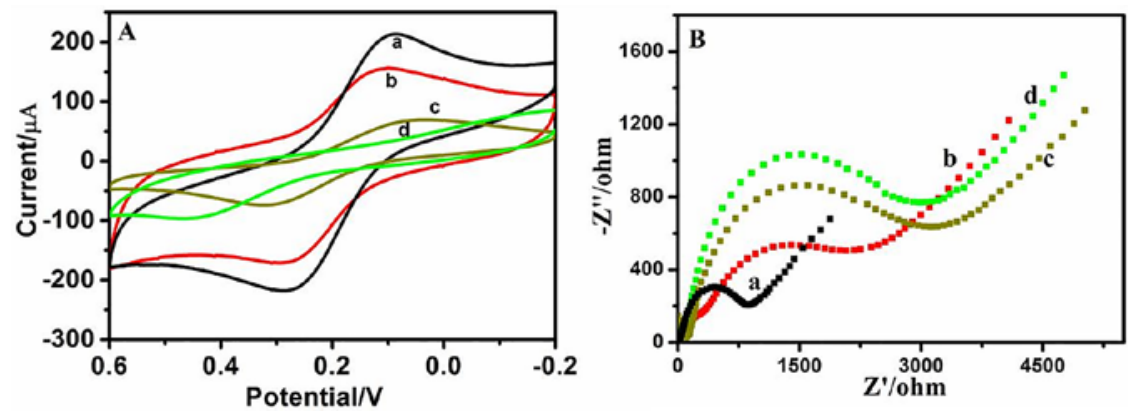

Fig. 5 (A) CVs and (B) Nyquist diagrams recorded at (a) 3D N-doped rGO/AuNPs, (b) 3D

N-doped rGO/AuNPs/ tetrahedral DNA, (c) miRNA-155 and (d) AuAgNR/Thi/F modified electrodes in $0.1 \mathrm{M} \mathrm{KCl}$ solution containing $2 \mathrm{mM} \mathrm{Fe}(\mathrm{CN})_{6}^{3-/ 4-}$ at a scan rate of $100 \mathrm{mV} \mathrm{s}^{-1}$
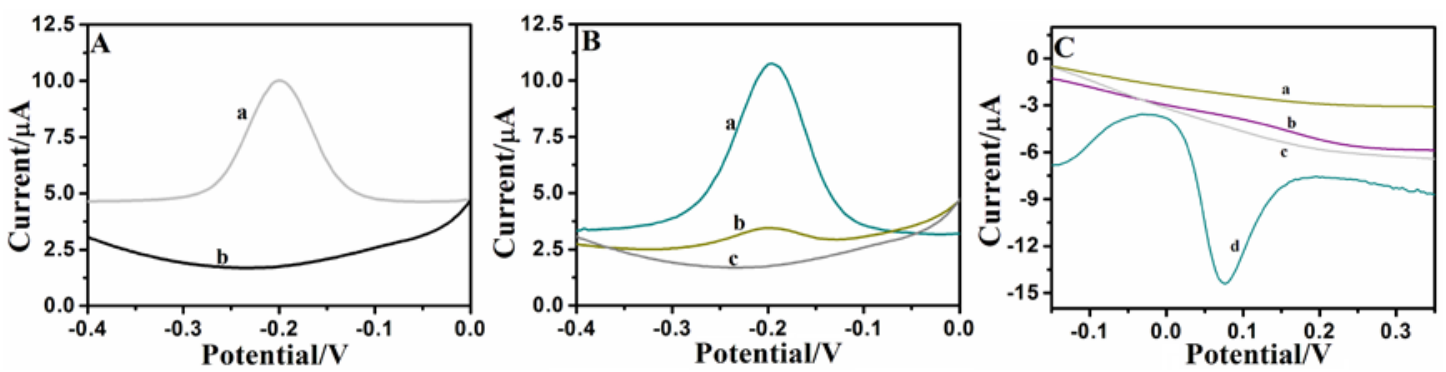

Fig. 6 DPV curves of the developed sensor incubated with $10 \mathrm{~nm}$ miRNA-155: (A) with tetrahedral DNA (a) and without tetrahedral DNA (b); (B) with AuAgNR/Thi/F (a), with AuAgNR/Thi (b), with AuAgNR/F (c); (C) LSV responses obtained at (a) 3D N-doped rGO/AuNPs (b) DNA tetrahedron modified electrode (c) after incubation with miRNA-155 and (d) after incubation with AuAgNR/ F

\subsection{Optimization of the experimental parameters towards biosensor}

To improve the performance of the biosensor, experimental conditions were 
optimized, including the reaction time of AuAgNR/Thi/F with miRNA-155, the hybridization temperature, the incubation time of miRNA-155, the effect of AuAgNR/Thi/F loading and the solution $\mathrm{pH}$.

\subsubsection{The reaction time of $\mathrm{AuAgNR} / \mathrm{Thi} / \mathrm{F}$ with miRNA-155}

Since the miRNA-155 detection was mainly based on monitoring the electrochemical signal change of Thi oxidation peak, before and after the specific binding of AuAgNR/Thi/F with miRNA-155, the undesired adsorption of AuAgNR/Thi/F on the electrode surface could increase background noise to affect the sensitivity. Therefore, to obtain an optimized adsorption condition, the interaction time between AuAgNR/Thi/F and miRNA-155 was investigated in the range of 10 to 60 min with the addition of $10^{-8} \mathrm{M}$ miRNA-155. As showed in Fig. 7A, the current response increased with prolonged interaction time, and tended to level off after 30 min, indicating a saturated binding of AuAgNR/Thi/F with miRNA-155.

\subsubsection{The effect of temperature}

Temperature is an important factor that can affect the hybridization dynamics of DNA. The interaction temperature of 3D N-doped rGO/AuNPs with tetrahedral DNA was investigated in the range of 20 to $50{ }^{\circ} \mathrm{C}$ with the addition of $10^{-8} \mathrm{M}$ miRNA-155. As shown in Fig. 7B, the signal response reached a maximum at $37{ }^{\circ} \mathrm{C}$, and then began to drop after that. Therefore, in our experiment, $37{ }^{\circ} \mathrm{C}$ was chosen as the optimal temperature in consideration of the bioactivity of tetrahedral DNA.

\subsubsection{The incubation time of miRNA-155}

The incubation time of miRNA-155 could directly affect the reaction with tetrahedral DNA. Fig. 7C shows the effect of different incubation times on the current of the biosensor. The signal output increased gradually along with increasing in incubation time, reaching a plateau after $2.0 \mathrm{~h}$. Longer incubation time did not further improve the response of the biosensor. Therefore, $2.0 \mathrm{~h}$ was chosen as the reaction of the AuAgNR/Thi/F.

\subsubsection{The effect of AuAgNR/Thi/F loading}

The amount of AuAgNR/Thi/F loading can affect the response current of this kind of the biosensor. Fig. 7D showed the response curves of the modified biosensor 
with different AuAgNR/Thi/F loading. With the increase of the loading, the response current increased gradually. The response of the modified electrode reached a plateau after adding $10 \mu \mathrm{L}$ of the AuAgNR/Thi/F to the system.

\subsubsection{The effect of $\mathrm{pH}$}

The $\mathrm{pH}$ of substrate solution might influence the catalyze activity of biosensor. The $\mathrm{pH}$ dependence of the amperometric response to $10^{-8} \mathrm{M}$ miRNA-155 was investigated over the $\mathrm{pH}$ values from 5.0 to 9.0 in the substrate PBS. As shown in Fig. 7E, the maximum amperometric response was achieved at $\mathrm{pH}$ 7.0. Thus, $\mathrm{PBS}$ at $\mathrm{pH}$ 7.0 was chosen for subsequent experiments.
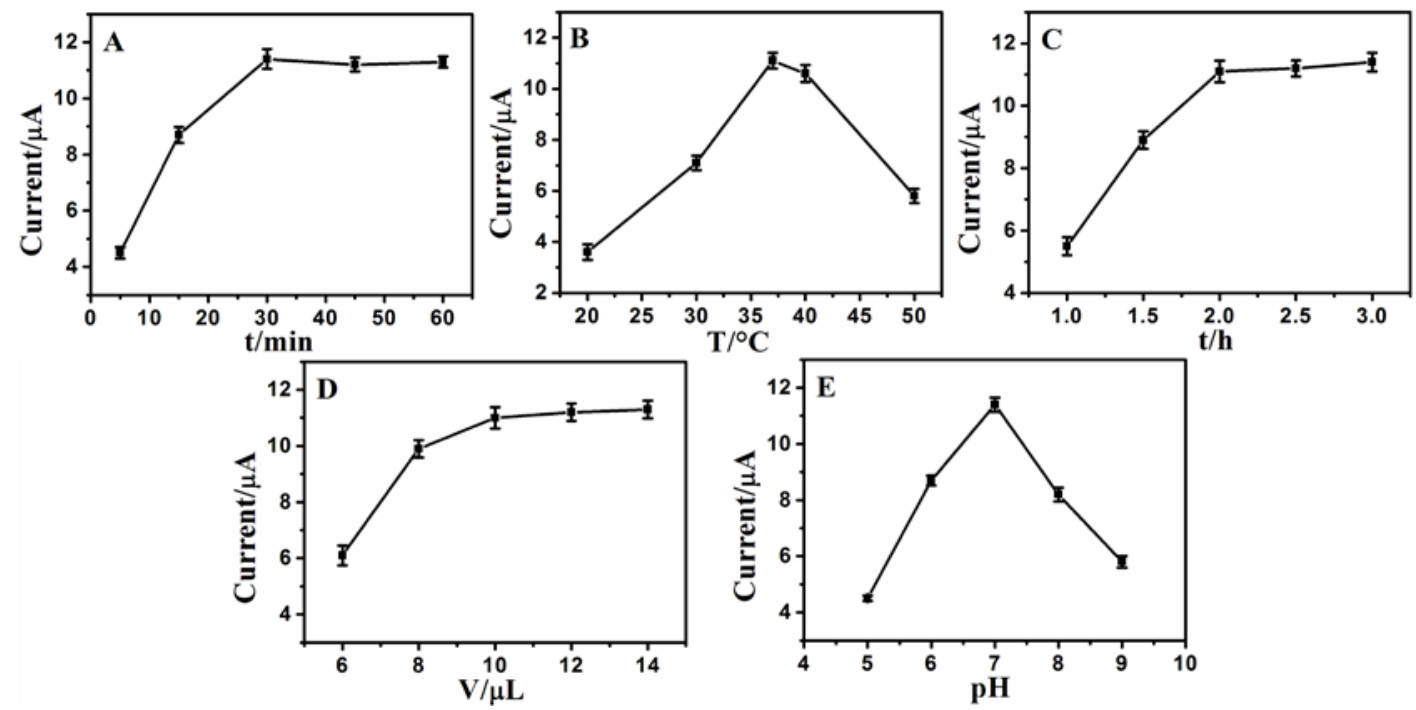

Fig. 7 (A) The reaction time of AuAgNR/Thi/F with miRNA-155, (B) the temperature, (C) the incubation time of miRNA-155,(D) the effect of AuAgNR/Thi/F loading and (E) $\mathrm{pH}$

\subsection{Analytical performance of biosensor}

The hybridization of miRNA-155 to tetrahedral DNA could lead to bind the F and tetrahedral DNA, the AuAgNR/Thi/F part could bind on the 3D N-doped rGO/AuNPs electrode, receiving the faradaic current response of Thi. This target induced new strands hybridization strategy was expected to give a much larger signal change magnitude than the target-induced conformational change, in which the electrochemical events were usually recorded based on the distance alteration between the redox species and the electrode surface. Under optimal conditions, the dynamic range of the developed miRNA-155 sensor was evaluated using AuAgNR/Thi/F adduct as reporter probe and the 3D N-doped rGO/AuNPs electrode as the sensing 
substrate. As shown in Fig. 8A, the DPV peak current increased with the increase of target miRNA-155 concentration. The response curve of the proposed biosensor for miRNA-155 showed a linear relationship between the reduction peak currents and the $\log$ of the analyte concentrations with a large dynamic range between $1 \times 10^{-11}$ and $1 \times 10^{-4} \mathrm{M}$, and a limit of detection of $1 \times 10^{-12} \mathrm{M}$. The deduced regression equation was $\mathrm{i}_{\mathrm{p}}=33.4+2.8 \log \left(\mathrm{C}_{\text {miRNA }}\right)$, with the relative coefficient of 0.992 . Such a high sensitivity was attributed to the signal amplification of the 3D N-doped rGO/AuNPs substrate as well as the assembled numerous Thi molecules for DPV response and the reducing background signal by the "signal-on" mode. Table 2 compared the analytical properties of the composite 3D N-doped rGO/AuNPs/tetrahedral DNA with other electrochemical biosensors in the literature. As comparison, our result exhibits a wider linear range and a lower LOD. Such a low LOD means that this detection strategy may have potential application in the human serum samples. This ultrasensitive detection of miRNA-155 may be attributed to 3D N-doped rGO/AuNPs and the AuAgNR adduct that can load more capture $\mathrm{F}$ and tetrahedral DNA molecules. In addition, 3D N-doped rGO/AuNPs film and AuAgNR may serve as conducting tunnels or wires to improve the conductivity of the sensing system.
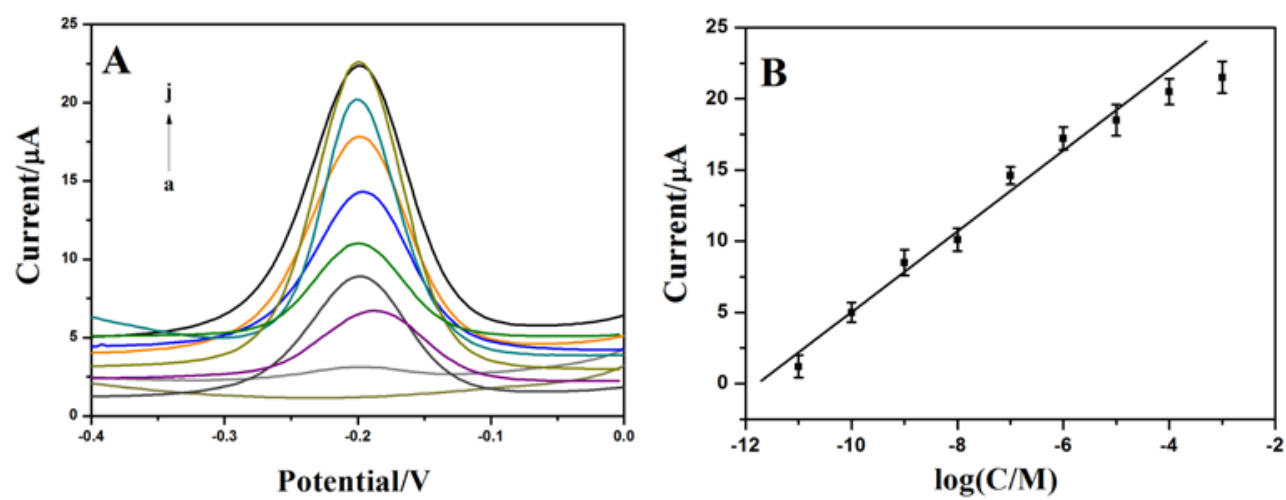

Fig. 8 (A) Typical DPV curves of the developed sensor in with different concentrations of miRNA-155 $\left(0,10^{-11}, 10^{-10}, 10^{-9}, 10^{-8}, 10^{-7}, 10^{-6}, 10^{-5}, 10^{-4}\right.$ and $\left.10^{-3} \mathrm{M}\right)(\mathrm{B})$ The linear relationship between the DPV peak current change and the logarithm of the concentrations of miRNA-155 Table 2 Comparison between the current method and other reported sensors for the detection of miRNA-155 


\begin{tabular}{cccc}
\hline Strategy & $\begin{array}{c}\text { Linear range } \\
(\mathrm{M})\end{array}$ & $\begin{array}{c}\text { Detection limit } \\
(\mathrm{pM})\end{array}$ & Refs. \\
\hline Graphene/gold nanoparticle & $1 \times 10^{-8} \sim 9.8 \times 10^{-7}$ & 3200 & {$[38]$} \\
Rolling circle amplification & $1 \times 10^{-11} \sim 4 \times 10^{-12}$ & 10 & {$[39]$} \\
3D DNA origami & $1 \times 10^{-10} \sim 1 \times 10^{-6}$ & 10 & {$[40]$} \\
enzymatic amplification & $2 \times 10^{-11} \sim 1 \times 10^{-9}$ & 9 & {$[41]$} \\
Tetrahedral DNA & $1 \times 10^{-12} \sim 1 \times 10^{-4}$ & 1 & This work \\
\hline
\end{tabular}

\subsection{Selectivity and reproducibility of the biosensor}

Selectivity is a critical criterion to evaluate a successful biosensor in target detection. In order to test the selectivity of the prepared biosensor for miRNA-155 detection, several possible interferences, such as miRNA-21, non-complementary sequence, single-base mismatched sequence and uric acid were investigated. As shown in Fig. 9, miRNA-155 showed the highest peak current among the several possible interferences studied and the fabricated biosensor showed negligible responses towards other analytes even at significantly high concentrations, demonstrating an excellent selectivity toward its target miRNA-155. To test the reproducibility, we fabricated five sensors and tested independently at the target concentration of $10 \mathrm{nM}$. The resulted relative standard deviation (RSD) was as low as $5.65 \%$. Additionally, the same biosensor was used to detect the same target miRNA-155 repetitively for five times, and the RSD was $1.21 \%$. These results suggested an encouraging reproducibility of the prepared miRNA-155 biosensors.

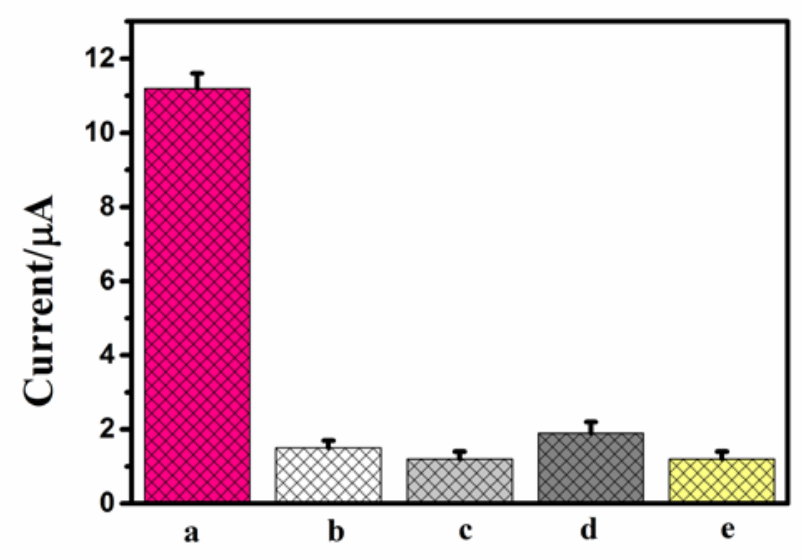


Fig. 9 The selectivity of the sensor hybridized to different miRNA sequences: E (a), G (b), H (c), I

(d) and uric acid (e). The concentration of miRNA-155 was $10 \mathrm{nM}$ and the concentration of the others was $500 \mathrm{nM}$, respectively

\subsection{Serum sample analysis}

With the rapid economic development in China, cancer has increasingly become an important health risk factor for people and the workers engaged in the miRNAs testing job. To investigate the practical application of this strategy in real sample analysis, we performed the miRNAs testing in serum samples. The testing samples were prepared by using the standard addition method by adding $0.1,1,10,100$ and $1000 \mathrm{nM}$ of miRNA-155 to serum samples. As shown in Table 3, a good recovery in the range of 94-105\% was observed, indicating that the proposed sensing platform can be potentially applied to monitor miRNA-155 in complex biological samples.

Table 3 Detection of miRNA-155 in serum samples with the proposed biosensor ( $\mathrm{n}=5$ )

\begin{tabular}{lccc}
\hline Sample (nM) & Added & Found (nM) & Recovery (\%) \\
\hline 1 & 0.1 & 0.105 & 105 \\
2 & 1 & 0.94 & 94 \\
3 & 10 & 9.55 & 95.5 \\
4 & 100 & 103 & 103 \\
5 & 1000 & 998 & 99.8 \\
\hline
\end{tabular}

\section{Conclusion}

We have demonstrated a new strategy for electrochemical detection of miRNA-155. The main advantages of the present biosensor contributed to two aspects. First, the fabricated 3D N-doped rGO/AuNPs film offers excellent electronic conductivity, large specific surface area for loading more tetrahedral DNA nanostructures-based biomolecular probe, which are remarkably better than those of present graphene composite materials. The thiolated DNA tetrahedral nanostructures binding to the AuNPs surface increases biomolecule target accessibility, and reduces the surface crowding effect. Second, compared with the reported electrostatic 
adsorption of Thi onto the capture DNA-functionalized electrode surface, the AuAgNR could only be immobilized on the electrode surface in the presence of miRNA-155, which decreased the blank signal significantly. Moreover, the method provides advantageous sensitivity, repeatability and stability comparing with present miRNA-155 sensors. These excellent properties can enable the real application in human serum sample, pave the way to liquid biopsies for early detection of cancer and relative diseases.

\section{Acknowledgment}

This work was supported financially by the National Natural Science Foundation of China (No. 2127369, 61704078), Tsinghua-Peking Joint Center for Life Sciences post-doctoral fund and the Natural Science Foundation of Jiangsu Province of China (grant no. BK20160990).

\section{References}

[1] A. Victor, The functions of animal microRNAs, Nature 431(2004)350-355.

[2] J. Lu, G. Getz, E.A. Miska, E. Alvarez-Saavedra, J. Lamb, D. Peck, A. Sweet-Cordero, B.L. Ebert, R.H. Mak, A.A. Ferrando, J.R. Downing, T. Jacks, H.R. Horvitz, T.R. Golub, MicroRNA expression profiles classify human cancers, Nature 435(2005)834-838.

[3] C. Jiang, X. Chen, M. Alattar, J. Wei, H. Liu, MicroRNAs in tumorigenesis, metastasis, diagnosis and prognosis of gastric cancer, Cancer Gene Ther. 22(2015)291-301.

[4] B.P. Lewis, Ih. Shih, M.W. Jones-Rhoades, D.P. Bartel, C.B. Burge, Prediction of mammalian microRNA targets, Cell 115(2003)787-798.

[5] J. Li, P. Lei, S. Ding, Y. Zhang, J. Yang, Q. Cheng, Y. Yan, An enzyme-free surface plasmon resonance biosensor for real-time detecting microRNA based on allosteric effect of mismatched catalytic hairpin assembly, Biosens. Bioelectron.77 (2016)435441.

[6] E.A. Hunt, D. Broyles, T. Head, S.K. Deo, MicroRNA detection: current technology and research strategies, Annu. Rev. Anal. Chem. 8(2015)217-237. 
[7] P. Ghosh, B. G. Roy, S.K. Mukhopadhyay and P. Banerjee, Recognition of fluoride anions at low ppm level inside living cells and from fluorosis affected tooth and saliva samples, RSC Adv., 5(2015) 27387-27392.

[8] A.R. Chowdhury, P. Ghosh, S. Paul, S. Bhuyan, J. C. Bose K, S. Mukhopadhyayd and P. Banerjee, A novel ditopic chemosensor for cadmium and fluoride and its possible application as a pH sensor. Anal. Methods, 9(2017)124-133.

[9] P. Ghosh and P. Banerjee, How paramagnetic and diamagnetic LMOCs detect picric acid from surface water and the intracellular environment: a combined experimental and DFT-D3 study, Phys. Chem. Chem. Phys., 18(2016)22805-22815.

[10] P. Ghosh, B. G.Roy, S. Jana, S. K. Mukhopadhyay and P. Banerjee, Colorimetric and fluorimetric response of Schiff base molecules towards fluoride anion, solution test kit fabrication, logical interpretations and DFT-D3 study, Phys. Chem. Chem. Phys., 17(2015)20288-20295.

[11] P. Ghosh, N.Kumar, S. K. Mukhopadhyay, P. Banerjee, Sensitive and fluorescent Schiff base chemosensor for pico molar level fluoride detection: In vitro study and mimic of logic gate function, Sensors and Actuators B: Chemical, 224( 2016)899-906. [12] P. Ghosh, P. Banerjee, Small molecular probe as selective tritopic sensor of $\mathrm{Al}^{3+}$, $\mathrm{F}^{-}$and TNP: Fabrication of portable prototype for onsite detection of explosive TNP, Analytica Chimica Acta, 965(2017)111-122.

[13] S. Azzouzi, W.C. Mak, K. Kor, A.P.F. Turner, M. Ben Ali, V. Beni, An integrated dual functional recognition/amplification bio-label for the one-step impedimetric detection of Micro-RNA-21, Biosens. Bioelectron. 92(2017)154-161.

[14] B. Zhang, B. Liu, G. Chen, D. Tang, Redox and catalysis 'all-in-one'infinite coordination polymer for electrochemical immunosensor of tumor markers, Biosens. Bioelectron. 64(2015)6-12.

[15] L. Liu, N. Xia, H. Liu, X. Kang, X. Liu, C. Xue, X. He, Highly sensitive and label-free electrochemical detection of microRNAs based on triple signal amplification of multifunctional gold nanoparticles, enzymes and redox-cycling reaction, Biosens. Bioelectron. 53(2014)399-405.

[16] Y. Zhang, Y.R. Yan, W.H. Chen, W. Cheng, S.Q. Li, X.J. Ding, D.D. Li, H. Wang, 
H.X. Ju, S.J. Ding, A simple electrochemical biosensor for highly sensitive and specific detection of microRNA based on mismatched catalytic hairpin assembly, Biosens. Bioelectron.68( 2015)343-349.

[17] P. Chen, J.J. Yang, S.S. Li, Z. Wang, T.Y. Xiao, Y.H. Qian, S.H. Yu, Hydrothermal synthesis of macroscopic nitrogen-doped graphene hydrogels for ultrafast supercapacitor, Nano Energy, 2(2013)249-256.

[18] Y. Lu, F. Zhang, T. Zhang, K. Leng, L. Zhang, X. Yang, Y. Ma, Y. Huang, Zhang, M., Chen, Y., Synthesis and supercapacitor performance studies of N-doped graphene materials using o-phenylenediamine as the double-N precursor, Carbon 63(2013)508-516.

[19] H.M. Jeong, J.W. Lee, W.H. Shin, Y.J. Choi, H.J. Shin, J.K. Kang, J.W. Choi, Nitrogen-doped graphene for high-performance ultracapacitors and the importance of nitrogen-doped sites at basal planes, Nano Letters, 11(2011)2472-2477.

[20] Z.S. Wu, S.B. Yang, Y. Sun, K. Parvez, X.L. Feng, K. Mullen, 3D Nitrogendoped graphene aerogel-Supported $\mathrm{Fe}_{3} \mathrm{O}_{4}$ nanoparticles as efficient electrocatalysts for the oxygen reduction reaction, J. Am. Chem. Soc. 134(2012) 9082-9085.

[21] X. Cao, N. Wang, S. Jia, L. Guo, K. Li, Bimetallic AuPt nanochains: Synthesis and their application in electrochemical immunosensor for the detection of carcinoembryonic antigen, Biosens. Bioelectron. 39(2013)226-230.

[22]Weilin Chen, Zhiqiang Zhu, Chang Yin, Yibao Li, Yi Liu, Yuting Zhang, Yulan Fan and Xiaolin Fan, Water-induced formation of a chiral phenylalanine derivative supramolecule, Physical Chemistry Chemical Physics, 20(2018) 4144-4148.

[23]J.-M. Lehn, Perspectives in supramolecular chemistry-from molecular recognition towards molecular information processing and self-organization. Angew. Chem., Int. Ed., 29(1990)1304-1319.

[24] M. Wang., B. Li, Q. Zhou, H. Yin, Y. Zhou, S. Ai, Label-free, ultrasensitive and electrochemical immunosensing platform for microRNA detection using Anti-DNA:

RNA hybrid antibody and enzymatic signal amplification, Electrochim. Acta 165(2015)130-135.

[25] X. Li, J. Guo, Q. Zhai, J. Xia, G. Yi, Ultrasensitive electrochemical biosensor for 
specific detection of DNA based on molecular beacon mediated circular strand displacement polymerization and hyperbranched rolling circle amplification, Anal. Chim. Acta 934(2016)52-58.

[26] T.K. Sau, C. J. Murphy, Seeded high yield synthesis of short Au nanorods in aqueous solution , Langmuir 20(2004)6414-6420.

[27] B. Nikoobakht, M.A. El-Sayed, Preparation and growth mechanism of gold nanorods (NRs) using seed-mediated growth method, Chem. Mater. 15(2003)1957-1962.

[28] J. Li, C.Y. Hong, S.X. Wu, H. Liang, L.P. Wang, G.M. Huang, X. Chen, H.H. Yang,D.H.Shangguan,W.H.Tan, Facile phase transfer and surface biofunctionalization of hydrophobic nanoparticles using Janus DNA tetrahedron nanostructures, J. Am. Chem. Soc. 137(2015)11210-11213.

[29] M.M Zhang, J.M. Xie, Q. Sun, Z.X. Yan, M. Chen, J.J. Jing, Enhanced electrocatalytic activity of high Pt-loadings on surface functionalized graphene nanosheets for methanol oxidation, Int. J. Hydrogen Energy 38( 2013)16402-16409. [30] W. He, H.J. Jiang, Y. Zhou, S.D. Yang, X.Z. Xue, Z.Q. Zou, X.G. Zhang, D. L. Aklins, H. Yang, An efficient reduction route for the production of Pd-Pt nanoparticles anchored on graphene nanosheets for use as durable oxygen reduction electrocatalysts, Carbon 50(2012)265-274.

[31] C.H. Tan, J. Cao, A.M. Khattak, F.-P. Cai, B. Jiang, G. Yang, S.-Q. Hu, High-performance tin oxide-nitrogen doped graphene aerogel hybrids as anode materials for lithium-ion batteries, J. Power Sources 270(2014)28-33.

[32] P. Su, H.L. Guo, S. Peng, Preparation of nitrogen-doped graphene and its supercapacitive properties, Acta Phys.-Chim. Sin. 28(2012)2745-2753.

[33] M.Z. Liu, P. Guyot-Sionnest, Synthesis and optical characterization of Au/Ag core/shell nanorods, J. Phys. Chem. B 108(2004)5882-5888.

[34] C.S. Ah, S.D. Hong, D.J. Jang, Preparation of Au core Ag shell nanorods and characterization of their surface plasmon resonances, J. Phys. Chem. B 105( 2001)7871-7873.

[35] M.F. Cardinal, B. Rodriguez-Gonzalez, R.A. Alvarez-Puebla, J. Perez-Juste, L.M. 
Liz-Marzan, Modulation of localized surface plasmons and SERS response in gold dumbbells through silver coating, J. Phys. Chem. C 114(2010) 10417-10423.

[36] R.-P. Liang, X.-C. Tian, P. Qiu, J.-D. Qiu, Multiplexed electrochemical detection of trypsin and chymotrypsin based on distinguishable signal nanoprobes, Anal. Chem. 86(2014)9256-9263.

[37] H.-L. Sun, M.-M. Xu, Q.-H. Guo, Y.-X. Yuan, L.-M. Shen, R.-A. Gu, J.-L. Yao, Surface enhanced Raman spectroscopic studies on magnetic $\mathrm{Fe}_{3} \mathrm{O}_{4} @ \mathrm{AuAg}$ alloy core-shell nanoparticle. Spectrochim. Acta. A 114 (2013) 579-585.

[38] H. Zhao, Y. Qu, F. Yuan, X. Quan, A visible and label-free colorimetric sensor for miRNA-21 detection based on peroxidase-like activity of graphene/gold- nanoparticle hybrids, Anal. Methods 8(2016)2005-2012.

[39] Y. Shi, H.Q. Luo, N.B. Li, A highly sensitive resonance Rayleigh scattering method to discriminate a parallel-stranded G-quadruplex from DNA with other topologies and structures, Chem. Commun.49(2013)6209-6211.

[40] S. Liu, W. Su, Z. Li, X. Ding, Electrochemical detection of lung cancer specific microRNAs using 3D DNA origami nanostructures, Biosens. Bioelectron. 71(2015)57-61.

[41] L. Cui, X. Lin, N. Lin, Y. Song, Z. Zhu, X. Chen, C.J. Yang, Graphene oxide-protected DNA probes for multiplex microRNA analysis in complex biological samples based on a cyclic enzymatic amplification method, Chem. Commun.48(2012) 194-196. 